\title{
Evaluating the Effect of Motion and Body Shape on the Perceived Sex of Virtual Characters
}

\author{
RACHEL MCDONNELL, SOPHIE JÖRG, FIONA NEWELL, and CAROL O'SULLIVAN \\ Trinity College Dublin \\ JESSICA K. HODGINS—Carnegie Mellon University
}

\begin{abstract}
In this paper, our aim is to determine factors that influence the perceived sex of virtual characters. In Experiment 1 , four different model types were used: highly realistic male and female models, an androgynous character, and a point light walker. Three different types of motion were applied to all models: motion captured male and female walks, and neutral synthetic walks. We found that both form and motion influence sex perception for these characters: for neutral synthetic motions, form determines perceived sex, whereas natural motion affects the perceived sex of both androgynous and realistic forms. These results indicate that the use of neutral walks is better than creating ambiguity by assigning an incongruent motion. In Experiment 2 we investigated further the influence of body shape and motion on realistic male and female models and found that adding stereotypical indicators of sex to the body shapes influenced sex perception. Also, that exaggerated female body shapes influences sex judgements more than exaggerated male shapes. These results have implications for variety and realism when simulating large crowds of virtual characters.
\end{abstract}

Categories and Subject Descriptors: I.3.7 [Computer Graphics]: Three Dimensional Graphics and Realism-Animation

General Terms: Experimentation, Human Factors

Additional Key Words and Phrases: Perception, graphics, motion capture

\section{ACM Reference Format:}

McDonnell, R., Jörg, S., Hodgins, J. K., Newell, F., and O’Sullivan C. 2009. Evaluating the effect of motion and body shape on the perceived sex of virtual characters. ACM Trans. Appl. Percpt. 5, 4, Article 20 (January 2009), 14 pages. DOI = 10.1145/ 1462048.1462051 http://doi.acm.org/10.1145/1462048.1462051

\section{INTRODUCTION}

Animated virtual humans are needed for many applications in entertainment, education, and science. Their movements can be created by artists, by programs (procedural animation), or recorded from real people using motion capture technology. Crowd simulation systems, in which thousands of virtual humans navigate realistic environments such as cities present a particular challenge. Crowds simulated with synthetic walking motions can lack personality, so motion captured data can be used to add realism (Figure 1).

In this paper, we investigate some factors that affect the perceived sex of walking virtual humans, with a view to increasing the realism of pedestrians in real-time crowd simulations. We cannot simulate

Authors' address: R. McDonnell, S. Jörg, F. Newell and C. O’Sullivan, Graphics Research Group \& Institute of Neuroscience, Trinity College Dublin; J. K. Hodgins, School of Computer Science, Carnegie Mellon University.

Permission to make digital or hard copies of part or all of this work for personal or classroom use is granted without fee provided that copies are not made or distributed for profit or direct commercial advantage and that copies show this notice on the first page or initial screen of a display along with the full citation. Copyrights for components of this work owned by others than ACM must be honored. Abstracting with credit is permitted. To copy otherwise, to republish, to post on servers, to redistribute to lists, or to use any component of this work in other works requires prior specific permission and/or a fee. Permissions may be requested from Publications Dept., ACM, Inc., 2 Penn Plaza, Suite 701, New York, NY 10121-0701 USA, fax +1 (212) 869-0481, or permissions@acm.org.

(c) 2009 ACM 1544-3558/2009/01-ART20 \$5.00 DOI 10.1145/1462048.1462051 http://doi.acm.org/10.1145/1462048.1462051 


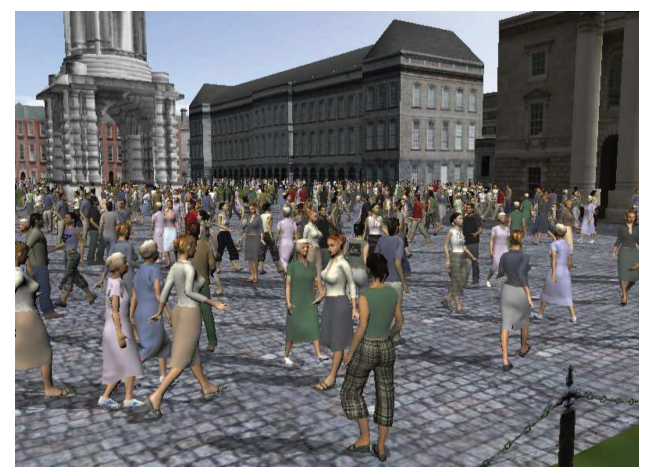

Fig. 1. Crowd simulation requires variety in colour, form, and motion [Dobbyn et al. 2006].
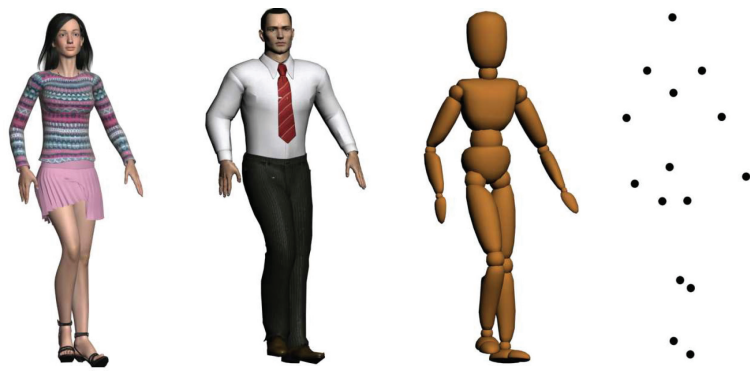

Fig. 2. Four model representations were animated with real female, real male, or synthetic neutral motions. From left to right: Woman model, Man model, Androgynous figure, and Point light walker.

everyone in a crowd with their own personal motion captured walk, as the more motions we use, the greater the demands on potentially limited computational and memory resources (e.g., a games console or hand-held device). Therefore, the challenge is to optimise quality and variety with the resources available. Specifically, we ask the question whether, if there is a clear visual indicator of sex (i.e., a highly realistic, unambiguously female or male model, as shown in Figure 2), will motion or form information dominate our perception of the sex of the character? If motion information alone always determines perceived sex, then we would always need to create templates of every different motion for both males and females. However, if we find that form dominates or that simulated neutral motions are as good as captured natural motions under some circumstances, then such duplication may not always be necessary. Perhaps some actors' walks can be equally effectively applied to both male and female models. Any of these results would allow us to create "canonical" motions to which variety could later be added, irrespective of the sex of the model.

\section{BACKGROUND}

People expect a virtual character to behave in a manner befitting its appearance and they find discrepancies in behaviour disturbing [Vinayagamoorthy et al. 2006]. Furthermore, people's perception of the sex of a human representation has been studied in the experimental psychology literature, with respect to human motion with minimal shape information and the effect of body shape. Only recently have the effects of both shape and motion been considered together. A note on terminology: sometimes gender is used in the literature to refer to the sex of a person, i.e., male or female. However, gender perception refers to the classification of the femininity or masculinity of a human, which is a different metric, e.g., a man can have a feminine walk but should still be classified as a male. 
Johansson $[1973 ; 1976]$ reported that natural motion, in the absence of any spatial information, is a sufficient cue to determine the sex of a walker. His 'point-light' displays were designed to separate biological motion information from other sources of information that are normally intermingled with the motion of a human, such as form or outline. He showed that 12 moving light points suffice to create a rich perception of a moving human figure within a very short space of time (200msec, or five frames of a movie). They also reported that the sex of the walker could be identified even when the number of lights was reduced to two on the ankles. However, it was later shown that this could have been mainly due to stride length [Kozlowski and Cutting 1978].

Many studies since have used Johansson's point-light displays to show that biological motion perception, an extreme example of sophisticated pattern analysis in the brain, extends even further, e.g., to recognising a particular walker [Cutting and Kozlowski 1977] or even one's own walking pattern [Beardsworth and Buckner 1981]. Kozlowski and Cutting [1977] explored some of the parameters of sex recognition without familiarity cues and showed that, while point-lights are sufficient for recognising the sex of a walker for dynamic sequences, static images were found to be insufficient, i.e., the human form cannot be identified when using static point-light stimuli alone. Troje [2002] implemented a classifier that could discriminate between male and female walkers. When comparing the behaviour of the classifier with human observers they found that humans rely on dynamic information more than structural when classifying a walk as male or female.

Mather and Murdoch [1994] found that using views which display sex-specific lateral body sway, rather than the sagittal (side) views of earlier studies, improved recognition accuracy. Pitting structural and dynamic cues against each other, their point light walkers were synthetically created with either a male or female torso or an androgynous structure. They applied male and female synthetic walks to the male and female body structures and an androgynous walk to the androgynous structure. They found that, even when looking at incongruent pairings of torso and walks, the motion was a more salient source of sex information than the body structure. However, altering point light walkers to display different body types may still leave uncertainty with respect to their sex, so perhaps it is not surprising that motion overrides shape in this case. Also, using synthetic motions that have exaggerated properties of male and female walks may not produce the same effects as natural motions.

Johnson and Tassinary [2005] studied the effects of both shape and motion on the perception of sex, gender, and attractiveness. Instead of using point light walkers which give little body shape information, they used silhouettes of human body shapes with varying waist to hip ratios, from exaggerated hourglass to tubular figures. Even though synthetic motion was restricted to two highly informative parameters (i.e., swagger for men and hip sway for women), they found that the shape of the character was more informative of the sex of the individual than its motion. They recently followed this up with a study in which they showed that both form and motion information, using these exaggerated feminine and masculine cues, contributed to participants' judgments of attractiveness [Johnson and Tassinary 2007].

In summary, previous work has shown that, when using exaggerated synthetic motion on point light walkers of differing shapes, motion was more dominant than shape information in sex perception. Conversely, when using synthetic motion on exaggerated male and female silhouettes, morphology was more influential than motion. In this paper, we describe two experiments where natural female and male motions, as well as synthetic neutral motions, were applied to very realistic male and female models (e.g., as may be found in modern computer graphics applications). In Experiment 1, we directly compare results with an androgynous shape and point light walker and also examine the effects of incongruent shape and motion combinations. In Experiment 2, we further investigate the effect of different male and female shapes in order to determine whether a scale of effect is evident or whether information about body shape interacts with motion information in the perception of the sex of the model. 


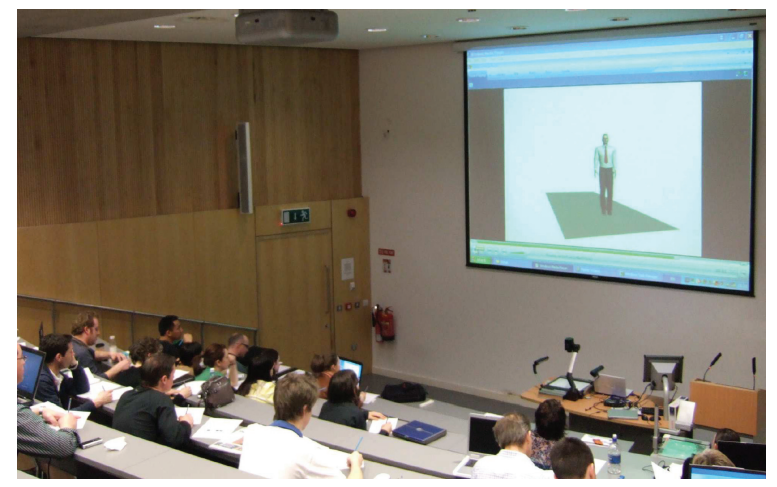

Fig. 3. Group participating in experiment.

\section{EXPERIMENT 1}

Forty-one participants (22M, 19F, aged 20 to 45 ) took part in this experiment to determine whether the motion or the shape/form of a virtual character is more salient for judgements of perceived sex of the model. All participants were naïve to the purpose of the experiment and had normal or corrected to normal vision. Participants (consisting of staff, students, and professionals from different educational backgrounds) viewed the stimuli on a large projected screen in a lecture theatre (see Figure 3). We used a two-way, repeated measures design and the conditions were motion type (3) and model type (4).

\subsection{Motion Type}

Six undergraduate students $(3 \mathrm{M}, 3 \mathrm{~F})$ volunteered to be motion captured, each in a separate session per actor. Since the walks were motion captured and, therefore, real we chose our volunteers randomly and did not try to ensure that their walks appeared particularly male or female. They were not informed as to the purpose of the motion capture session which was conducted using our 10 camera Vicon optical system, using 41 markers placed according to the Vicon human template. A curved path was drawn on the floor in the capture area and they were asked to walk naturally up and down this path, which they did about 20 times. We captured some of the walks without their knowledge to ensure they were walking naturally, then applied the motion capture data to characters in 3D Studio Max and kept one natural walk per actor (see video at http://isg.cs.tcd.ie/mcdonner/TAP/). Since it would not be possible to capture a truly neutral walk, we synthetically generated three different neutral walk motions, using 3D Studio Max's footstep modifier. This modifier allows the automatic generation of synthetic walk motions using a kinematic solver. These walks are neutral as they have neither male nor female characteristics (such as hip sway or shoulder movement). The distance between footplants was altered in order to create three unique neutral walks.

\subsection{Model Type}

Four different models were used to display the different motions (Figure 2): highly detailed woman and man models of approximately 35000 polygons each, an androgynous character, and a point light walker. The woman and man were chosen as typical characters that would be used in a computer simulation of natural crowds. The androgynous figure was chosen as it did not appear particularly male or female in shape and so could serve as a control. The point light walker was generated from a generic neutral skeleton and so contained minimal shape information. We included this representation both as a baseline and to compare with the results of the earlier studies described above. 

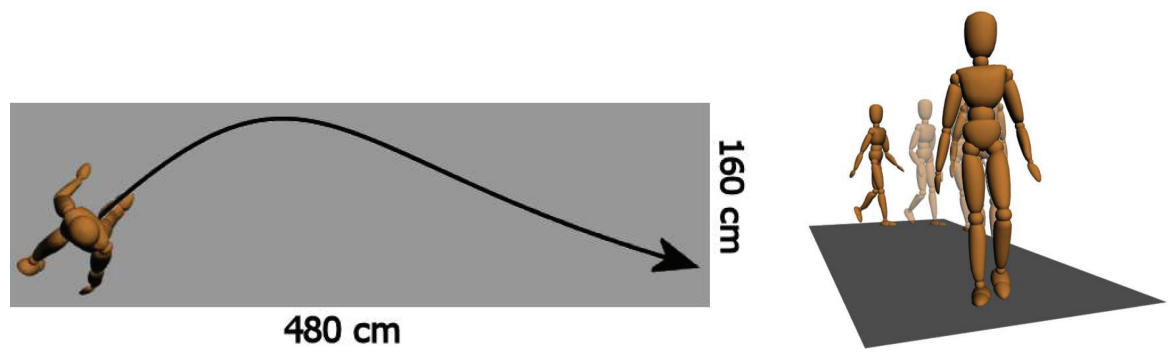

Fig. 4. (L) Walker's path, (R) Camera view.

A grey ground plane was added to the scene and the camera was placed so that the majority of the walk was in a straight line coming towards the camera (Figure 4), since Halevina and Troje found the frontal view facilitates sex classification [2007]. Each of the different motion types (3) from each of the actors (3) were applied to each of the model types (4), with two repetitions for each condition resulting in a total of 72 movies, each presented for a duration of approximately 3.5 seconds long (the length of time it took the actor to walk from one end of the motion capture area to the other).

The movies were sorted randomly in two different playlists and two groups of participants each viewed one of the sets of 72 movies on a large projected display ${ }^{1}$ (Figure 3 ). They were told to take both motion and form/shape into account when making judgements about the sex of the character and they marked their selections on an answer sheet. Participants sat near to one another, but were not allowed to discuss their decisions. Each movie was followed by a four-second blank screen, during which participants categorised the character they just saw on a five-point scale of 1: very male, 2: male, 3 : ambiguous, 4: female, or 5: very female. The number of the next stimulus was then displayed for two seconds with an accompanying alerting sound.

\subsection{Results}

Before beginning the experiment, participants were shown a static image of the androgynous figure and asked to rate it on the same five-point scale. As it was considered to be ambiguous by almost all participants we concluded that, based on shape/form information alone, the figure truly appeared androgynous.

We averaged participants' ratings over the two repetitions and three actors for each combination of walk and model type. We first found that the sex of the participants had no effect, as in [Kozlowski and Cutting 1977; Johnson and Tassinary 2005]. A two factor ANalysis Of VAriance (ANOVA) [Howell 1999] with repeated measures showed a main effect of walk type $\left(F_{2,80}=130, p<0.001\right)$. Post-hoc analysis was then performed using a standard Newman-Keuls test for pairwise comparisons among means. In Figure 5 (left) it can be seen that, as expected, female walks were rated overall as female, neutral walks as neutral, and male walks as male ( $p<0.001$ in all cases). There was also a main effect of model type $\left(F_{3,120}=24, p<0.001\right)$. Figure $5(\mathrm{R})$ shows that the man was considered significantly more male than the woman or the androgynous figure, while the point walker was considered significantly less male than the man and less female than the woman or the androgynous figure ( $p<0.001$ in all cases). There was also an interaction between model type and walk type $\left(F_{6,240}=25, p<0.001\right)$.

Figure 6 shows that male walks on the woman affect the sex judgement of the female form such that it is now rated as ambiguous. A similar effect is observed when a female walk is applied to a male shape.

\footnotetext{
${ }^{1}$ Similar group experiments were conducted with only a monitor at the front of the lecture theatre [Kozlowski and Cutting 1977]. Even though viewing angle differed significantly between participants, recognition performance was still well above chance. Our larger display ensured more consistent viewing angles.
} 

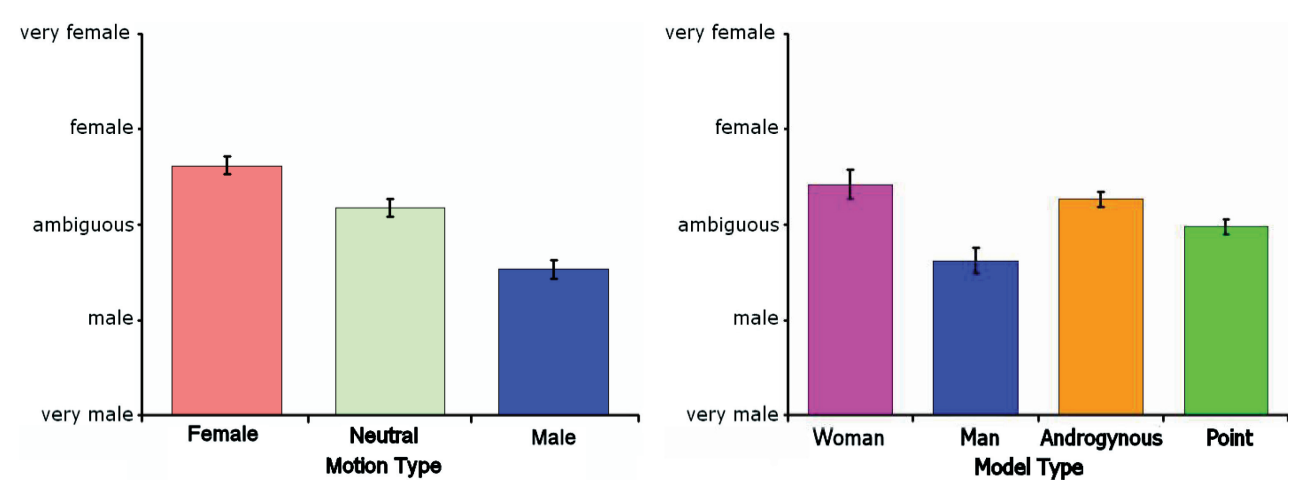

Fig. 5. Main effects of (L) walk type (averaged over all models), (R) model type (averaged over all motions)-with standard errors.

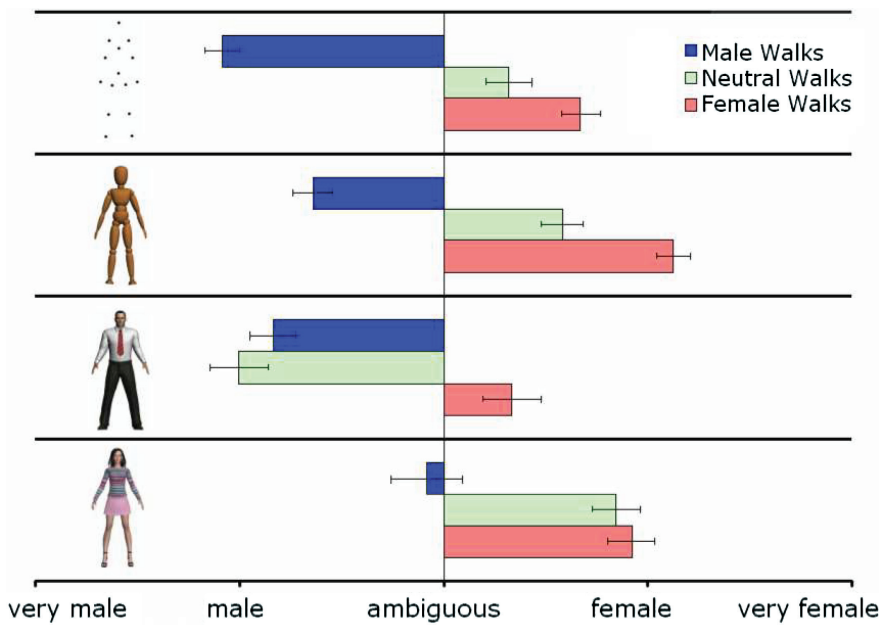

Fig. 6. The interacting effects of model and walk type.

This implies that applying motion captured from actors of the opposite sex to the character will produce confusing or unsatisfactory results in general. Interestingly, neutral walks were considered male when viewed on the man and female when viewed on the woman. This implies that for neutral walks, the shape of the character takes precedence over the motion in determining the sex of the character. This result has implications for computer graphics applications where resources are limited, as reusing the same neutral walks on male and female characters would appear to produce the desired effect.

As illustrated in Figure 6, there were three significantly different ratings for the androgynous figure for male, neutral, and female walks $(p<0.001)$. This implies that for a character with androgynous appearance, the motion information is most important when determining the sex (as without motion, the androgynous figure was consistently rated to be ambiguous). Replicating the results of previous studies, we showed that participants were able to determine the sex of the walker using point light walkers with good accuracy.

We then looked more closely at the ratings for each of the motion captured walks. Female walks 1 and 2 (F1, F2) were consistently rated as female for all model types (see Figures 7 and 8). However, F3 was consistently rated as either ambiguous (for the woman and the androgynous figure) or slightly male (for the man and the point walker). We noticed that the female actor that performed F3 did not move her 
Average classification for all walkers

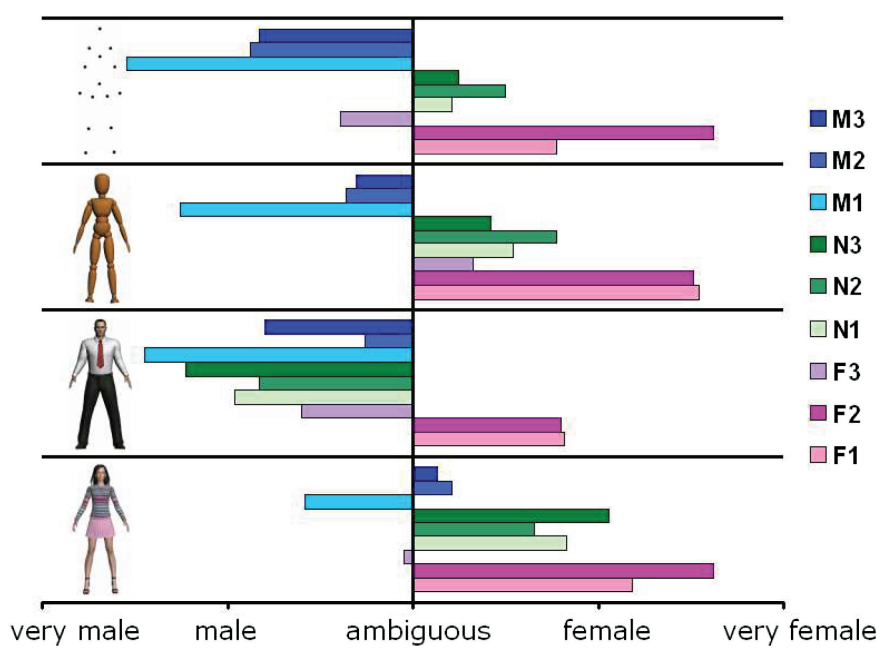

Fig. 7. Classifications of individual walkers for each model type (Female walks F1-F3, Males M1-M3, Neutral N1-N3).

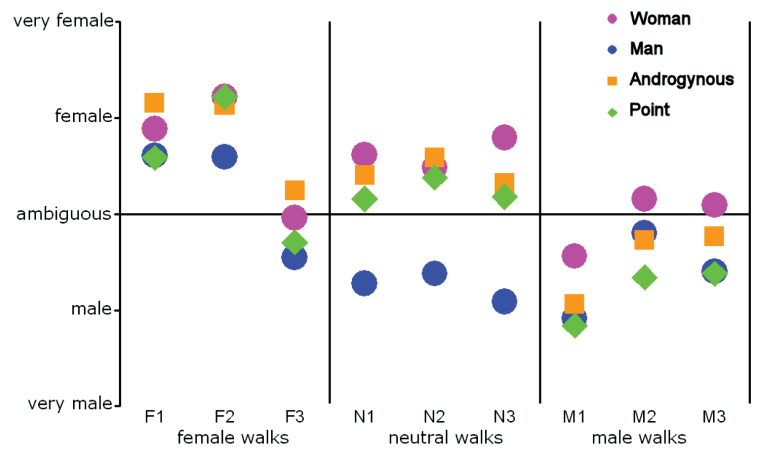

Fig. 8. Classifications of individual walkers for each model type (Female walks F1-F3, Males M1-M3, Neutral N1-N3).

hips much, but swung her arms quite a lot and her natural walk was slightly faster than the other two females. This result implies that care should be taken when choosing an actor, as misinterpretations can occur. This was also the case in [Kozlowski and Cutting 1977] where they found that one of their female walks was more difficult to rate than the others.

The three neutral walks were ranked similarly to each other for each of the model types. Also, a similar trend across the three different male walks was found for each model type, with M1 considered significantly more male than the other two male walks ( $p<0.001$ in all cases). For the man, the motion of M3 was considered more masculine than M2. The actor that performed M2 did appear to have more hip sway than the other actors when we were capturing the session, therefore it is unsurprising that his walk was considered less masculine. However, it is interesting that this was most evident on the male model.

In order to judge overall sex recognition accuracy for the motions and to see if our results are compatible with earlier point-light walker experiments, such as Kozlowski and Cutting's [1977], we converted our scaled judgements of sex into percentage correct values (with 4 or 5 being female, 1 or 2 male, 

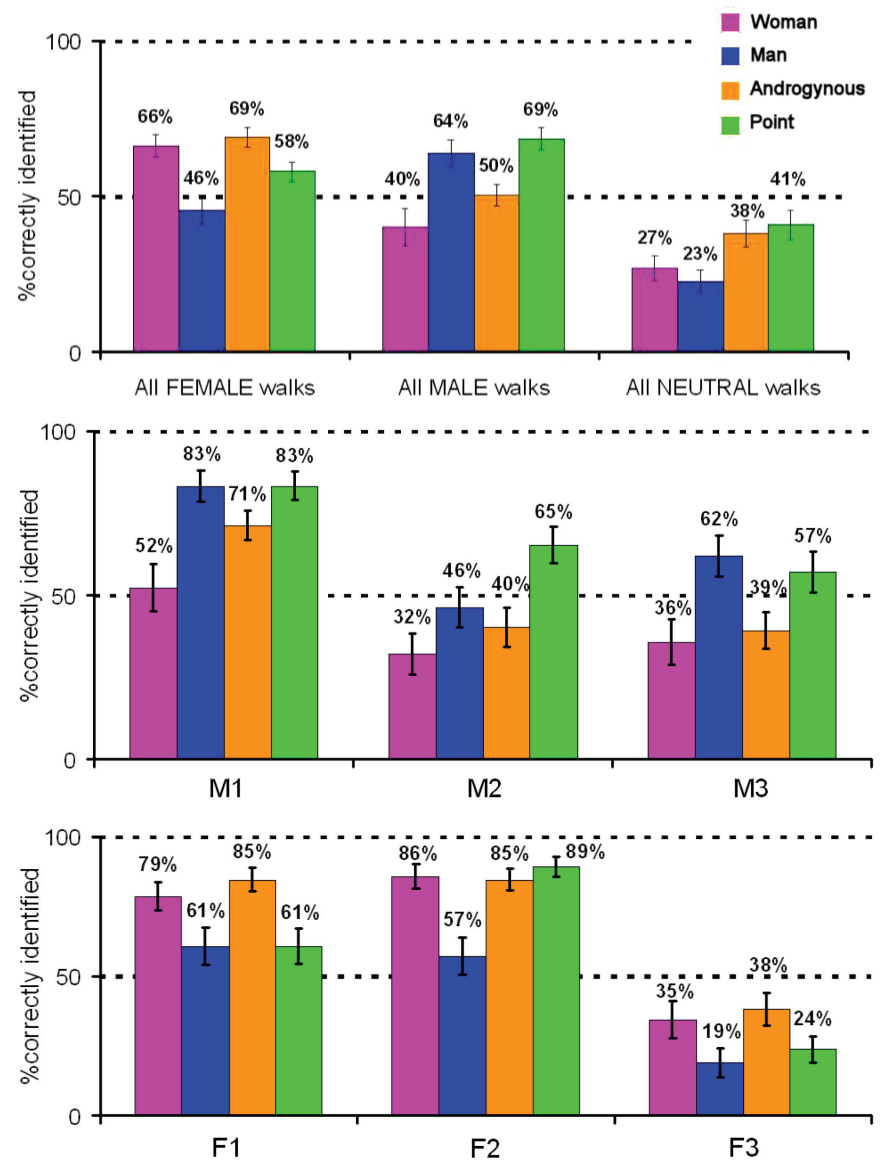

Fig. 9. Identification accuracy for recognition of walker sex (top) overall; (middle) for the three male walks; (bottom) for the three female walks.

and 3 neutral). The results are shown in Figure 9. For the point walker, 69\% of male walks were correctly identified, which is consistent with the earlier study where it was found that recognition accuracy hovered around $70 \%$. However, a significantly lower percentage (58\%) of females were correctly identified than males $\left(F_{82,1}=4.77, p<0.05\right)$. This can be explained by the fact that F3 was consistently misclassified as male and had significantly different results from the other female walks for all models.

Noteworthy too is the fact that the accuracy for female walks on the male character is equally poor to that for male walks on the female character (both below chance), while the congruent motion on both is rated equally high at about $65 \%$. Particularly interesting too are the results for the neutral walks, where well under $50 \%$ of them were actually classified as neutral for any of the models. In particular, only about $25 \%$ of the neutral walks on the realistic characters were perceived to be ambiguous. Closer examination reveals that $62 \%$ of neutral walks were classified as female on the woman, but only $11 \%$ as male $\left(F_{82,1}=14.22, p<0.0005\right)$, with an equal and opposite effect on the man, with $65 \%$ classified as male and $13 \%$ as female $\left(F_{82,1}=8.19, p<0.005\right)$. 

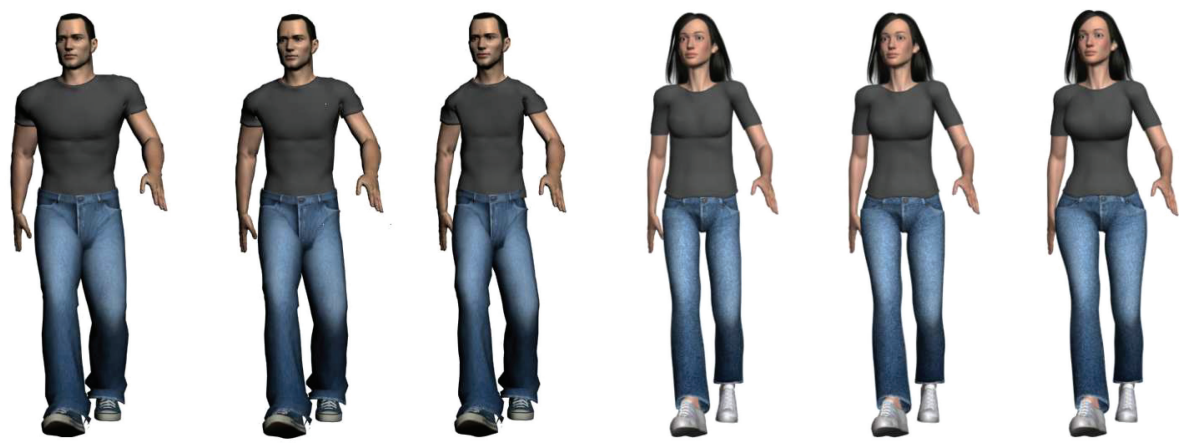

Fig. 10. Six body shapes used in Experiment 2: Man 3, Man 2, Man 1, Woman 1, Woman 2, Woman 3

\section{EXPERIMENT 2}

In Experiment 1, we chose only two models to represent male and female appearance. In this experiment, we investigated the effect of motion on different male and female shapes to determine if additional indicators of sex in the shape will change the overall perception of the sex of the character.

\subsection{Body Shapes}

We used the same man and woman models as in the previous experiment. However, this time they both wore neutral clothing (jeans and a grey t-shirt). Using 3D Studio Max, we manipulated the polygons of the woman model in order to create a very exaggerated female body shape. We increased the size of her breasts and hips, in order to give her an hour glass figure with a large waist-to-hip ratio (Woman 3). We applied equivalent alterations to the man model by increasing his shoulder, waist, and neck width to create a more exaggerated male body shape (Man 3).

Next, we created a more androgynous version of the female by decreasing her waist-to-hip ratio and breast size (Woman 1), and of the man by decreasing his chest, neck, and waist size and the distance between his shoulders (Man 1). Finally, we created a morphed female body shape (Woman 2), which was a half way morph between Woman 1 and Woman 3. Similarly, we created a halfway morph between Man 1 and Man 3 (Man 2)-see Figure 10. All bodies were created based on our own estimates of physiological plausibility which were consistent with standard physical proportions. In participant feedback, no comments regarding physical abnormalities were made.

\subsection{Procedure}

Each of the nine different motion types from the previous experiment (three male, three neutral, and three female) were applied to each of the body shapes (6), with two repetitions for each condition, resulting in a total of 108 movies. Three random playlists, each including the 108 movies, were created and three groups of participants each viewed one of the playlists on a large projected display. Thirtyeight participants took part in this experiment (33M, 5F). All participants were naïve to the purpose of the experiment and had normal or corrected to normal vision. Participants consisted mainly of computer science staff and students. As before, they were told to take both motion and form into account, and they categorised the character on a five-point scale which ranged from very male to very female.

\subsection{Results}

As before, we averaged participants' ratings over the two repetitions and three actors for each combination of walk and body shapes. A two factor ANOVA with repeated measures showed a main effect of walk type $\left(F_{2,74}=94.65, p<0.001\right)$ —see Figure 11(L). Standard Newman-Keuls tests for comparisons 

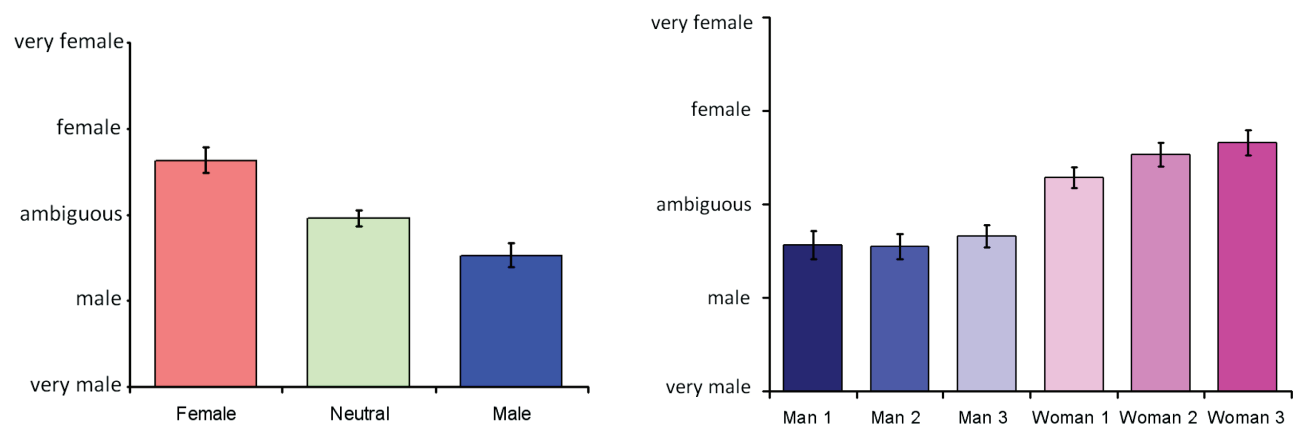

Fig. 11. Main effects of (L) walk type (averaged over all models), (R) body shape (averaged over all motions).

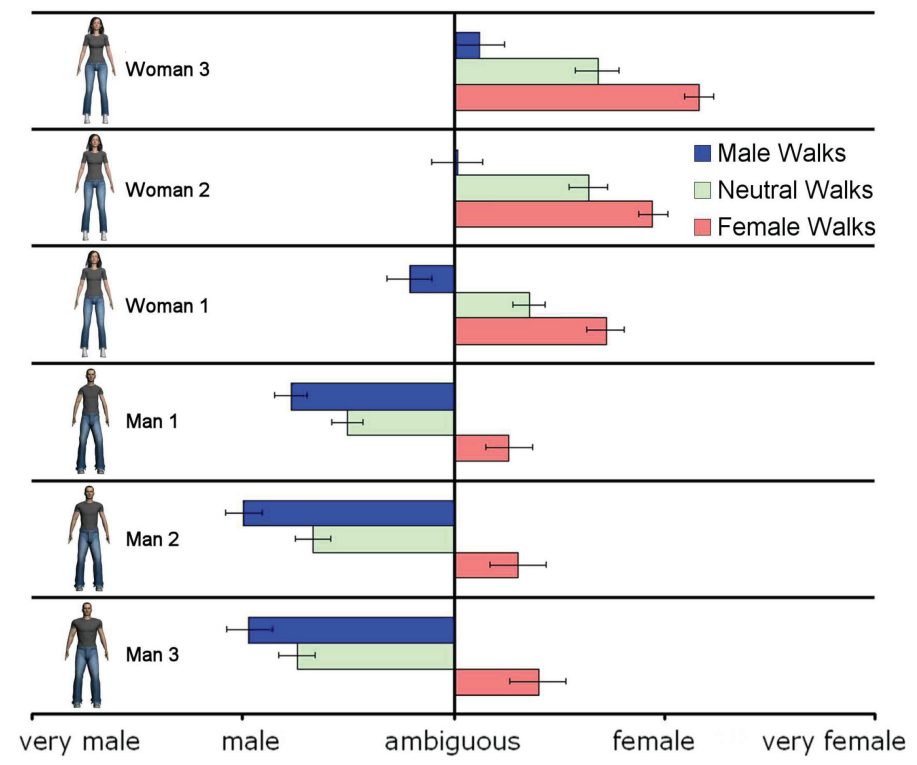

Fig. 12. The interacting effects of shape and walk type.

among means showed us that, as before, female walks were rated overall as female, neutral walks as neutral, and male walks as male ( $p<0.001$ in all cases). There was also a main effect of body shape $\left(F_{5,185}=39.73, p<0.001\right)$. Figure $11(\mathrm{R})$ shows that the three man shapes were considered significantly more male than the three woman shapes ( $p<0.001$ in all cases). Surprisingly, there was no statistical significance between participants' ratings of Man 1, Man 2, or Man 3. However, there was a difference between ratings of Woman 1 and Woman 2, and Woman 1 and Woman 3, with Woman 1 being rated as less female in both cases $(p<0.04$ and $p<0.004$ resp.). There was no difference in perception between Woman 2 and Woman 3.

There was also a significant interaction $\left(F_{10,370}=10.28, p<0.001\right)$ between walk type and body shape (see Figure 12). Therefore, a post-hoc analysis was conducted using Newman-Keuls tests for comparison among means.

Perhaps the most obvious result that may have contributed to this interaction effect is that the male walks did not affect the perception of sex in the female shapes to the same extent that the female walk affected the perception of the sex of the male models. For all the male models, applying a female 
walk biased the perception of the sex of the shape of the male. But this did not occur for two-thirds of the female shapes. This suggests that female form is slightly more salient than male form in sex judgements (although a male walk clearly reduced the number of 'female' judgements of all the female shapes, so motion was still a powerful cue).

We found that for all walk types, body shapes Man 3 and Man 2 were not significantly different from each other. This implies that the difference between these two shape types had no effect on judgements of sex, irrespective of motion type. Perhaps it was the case that Man 2 had already sufficient indicators of sex that the extra information for Man 3 did not change our perception. Sex judgements for Man 1 were significantly different from both Man 2 and Man 3 for male and neutral walks but not for female walks.

Woman 1 was considered less female than both Woman 2 and Woman 3 for all walk types. Woman 2 was considered less female than Woman 3 for only female walks. This implies that it was only for the female walks that the extra female shape characteristics had an effect. Perhaps this was due to the hip sway in the female walks being exaggerated by the increased waist-to-hip ratio.

As in Experiment 1, male walks on the Woman body shapes rendered sex judgements as more ambiguous, as did the female walks on the Man body shapes. Neutral walks were still considered female on the Woman characters, but in this experiment we can see that they are rated significantly less female than the female walks. Similarly, the neutral walks on the Man characters were considered male, but again less male than the male walks. This is an interesting result, as it shows that neutral walks could be used for congruency, as the shape of the character is dominant, but there is a point at which extra indicators of sex in the shape have no effect.

In Experiment 1, we found no difference between the ratings of motion captured male and neutral walks for the man model. Similarly for the woman model, there was no difference in ratings between the female and neutral walks. Perhaps this was due to the clothing worn by the models in Experiment 1 having extra indicators of sex than the neutral clothing in Experiment 2. More likely is the case that, in Experiment 1 participants used the same rating scale as in Experiment 2 to cover a wider range of models. There were no androgynous characters in Experiment 2 as this experiment focused more on the properties of the man and woman models. The presence of these characters in Experiment 1 could have influenced the participants to rate the neutral walks on the man and woman as more male or female than they might have otherwise. We feel that the results for the neutral motions on man and woman models in Experiment 2 could be considered more accurate, since they could not have been influenced by other model types.

Finally, we looked more closely at the ratings for each of the motion captured walks (see Figure 13). Female walks 1 and 2 (F1, F2) were consistently rated as female for all body shapes. In Experiment 1 , F3 was consistently rated as either ambiguous or slightly male. The same was true in this experiment, except that for the three Woman characters, increasingly more 'female' judgements were made, the more female the body shape. This suggests that perhaps the female characteristics of the walk were brought out by the exaggerated female shape.

As before, the three neutral walks were ranked similarly to each other for each of the model types. Similar effects were also found for two of the different male walks (M2 and M3) for each body shape. Interesting to note is how M1 was considered significantly less male on Man 1 than on Man 2 or Man 3 ( $p$ < 0.001). M1 was the walk judged as the most male in the previous experiment so it is interesting how the least male shape influenced the overall perception of the character to appear less male.

In this experiment, we found that both shape and motion had an effect on our perception of sex. For the three Woman body shapes, the female walks were judged as increasingly female the more female the shapes became. The neutral walks were affected by shape also, but Woman 2 and 3 were judged as equally female, implying that more exaggerated female shapes had no effect on the perceived sex when 


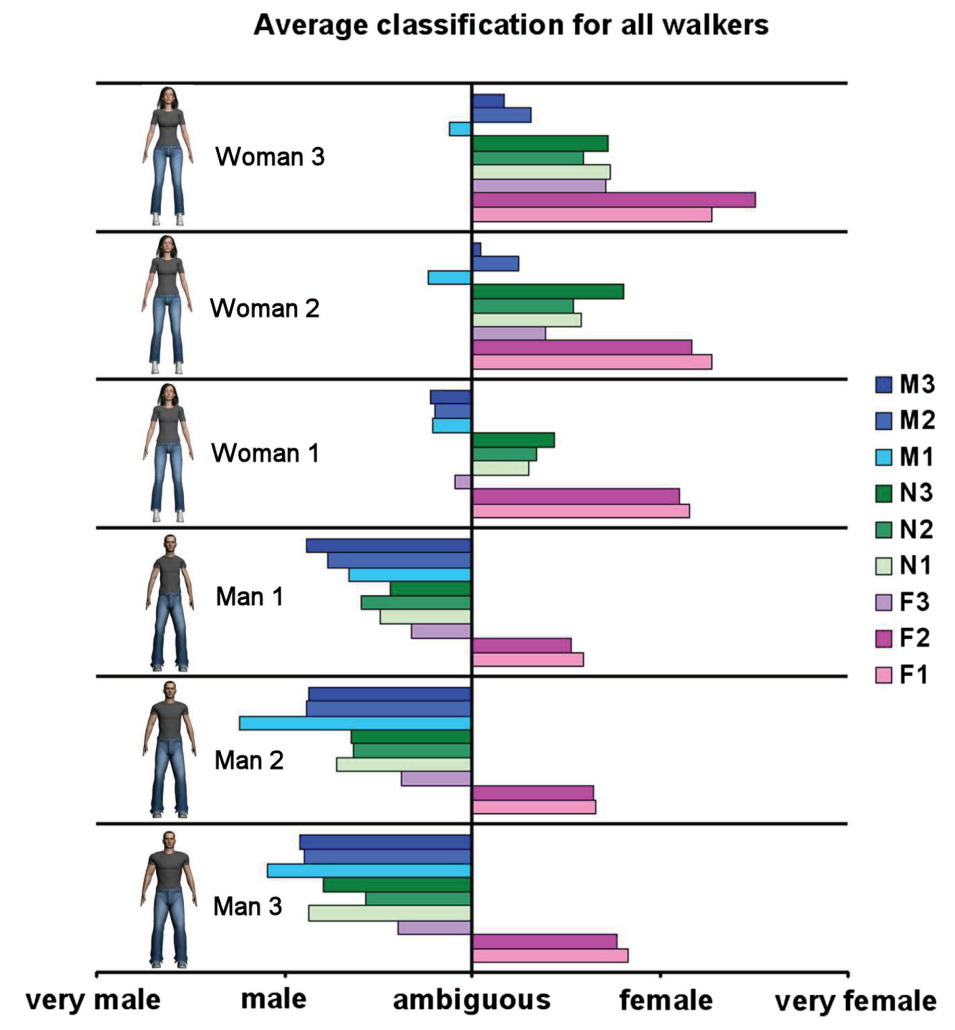

Fig. 13. Average classifications of individual walkers on body shape.

coupled with neutral walks. For the Man models, an increase in male shape between Man 1 and 2 (but not between 2 and 3) increased the number of male judgements when coupled with neutral and male walks, implying that extra male information on the Man model did not have an effect past Man 2.

This plateau for Man 2 for male and neutral walks may have been due to the fact that a large percentage of participants were male in this experiment and perhaps had more sensitivity towards female body shapes. To test this theory, we ran the experiment on a further 12 females and combined these results with the original five females, resulting in a total of 17 female participants. We randomly chose the results of 17 of the male participants and compared them to the female results using an ANOVA, where the repeated measure factors were model type and motion type, and between subjects factor was sex of participant. We found no main effect of the sex of the participant. We also found no interaction between participant sex and walk type. However, we did find a significant interaction $(p<0.001)$ between sex of participant and model type. On closer inspection, this interaction was due to the man models being rated as slightly more male overall and the woman models as slightly more female by the female participants. However, there were no differences between the ratings of the female and male participants for the three man or the three woman models. This indicated that the plateau at Man 2 for male and neutral walks may have been due to some other factor such as the model that we chose. What this indicates is that, despite [Kozlowski and Cutting 1977; Johnson and Tassinary 2005] and the results of Experiment 1, there is some evidence that there may be slight differences in perception of body types between males and females. 


\section{CONCLUSIONS}

In this paper we have identified some of the factors that influence perception of the sex of virtual characters. Unlike previous work, we found that both the appearance and the motion of the character influence perceived sex. We also found that for walk motions with no male or female characteristics, it is the appearance of the character that will dominate our perception of the character's sex, whereas it is the motion that dominates our perception for characters with an androgynous appearance.

This information will be useful in computer graphics applications, as it is now clear that in order to have a convincing male/female character, you must apply a female walk to a female character and a male walk to a male character. This implies that consistency is highly important when designing a virtual character. As discussed in [Vinayagamoorthy et al. 2006], people expect virtual characters to behave in a manner befitting its appearance, and the results of our study confirm this. However, care must be taken when choosing an actor, as we found some misinterpretations. From our results, it is certain that you cannot apply a female walk to a male character, or vice versa, as viewers will judge this conflicting information to be ambiguous.

A simple solution would be to reduce memory costs by using neutral walks, as overall they will look female on a female character, and male on a male character, but less so than the motion capture. In Experiment 2, we found that adding additional indicators of sex in the shape of the character increases how male or female a neutral walk is considered, but only up to a point. Also, we do not know how natural the participants found these animations and would like to investigate in future studies whether or not they found these motions to be natural (we have some implicit evidence that they did, however, as they had the option to categorise these walks as ambiguous, which they did for the other models). We may need to add stereotypical characteristics such as hip sway and shoulder movement to increase motion believability [Cutting 1978]. Therefore, a comparison of synthetic and natural walk motions could also be beneficial. Something akin to a "Turing test" could be appropriate here, where people would indicate whether a walking motion was real or synthetic.

It would be interesting to see if our results are replicated with other motions besides walking. Perhaps it is also the case for gestures; that male actors performing certain stereotypical gestures (e.g., woodcutting or boxing) will always be considered male. However, this may not be the case for unfamiliar motions such as those typically used in games. Perhaps it would be possible to reuse unfamiliar motions (such as shooting and kicking) from single actors on multiple game characters (both male and female). Furthermore, highly dynamic motions have less freedom for style than walking (because they are closer to the energy limit of the human body). Therefore, such motions may naturally be more neutral. However, the dynamics of the actor will play more of a role in such actions, so the properties of male/female style that are linked to the kinematics and dynamics of the body (e.g., wider hip spacing, smaller size, lower moment of inertia in females) could actually cause the differences to be more obvious in dynamic motions. Such issues merit further investigation.

\section{REFERENCES}

Beardsworth, T. And Buckner, T. 1981. The ability to recognize oneself from a video recording of ones movements without seeing ones body. Bulletin of the Psychonomic Society 18, 1, 19-22.

CutTing, J. 1978. Generation of synthetic male and female walkers through manipulation of a biomechanical invariant. Perception 7, 4, 393-403.

Cutting, J. AND Kozlowski, L. 1977. Recognizing friends by their walk: Gait perception without familiarity cues. Bulletin of the Psychonomic Society 9, 5, 353-356.

Dobbyn, S., McDonnell, R., Kavan, L., Collins, S., and O’Sullivan, C. 2006. Clothing the masses: Real-time clothed crowds with variation. In Eurographics Short Papers. 103-106.

Halevina, A. and Troje, N. 2007. Sex-classification of point-light walkers: Viewpoint, structure, kinematics. In Poster Presented at Vision Science Society Meeting, Sarasota, FL. 
Howell, D. 1999. Fundamental Statistics for the Behavioral Sciences. Duxbury Press.

Johansson, G. 1973. Visual perception of biological motion and a model for its analysis. Perception and Psychophysics 14, 2 , 201-211.

Johansson, G. 1976. Spatio-temporal differentiation and integration in visual motion perception. Psychological Research 38, 379-393.

Johnson, K. L. and Tassinary, L. G. 2005. Perceiving sex directly and indirectly: Meaning in motion and morphology. Psychological Science 16, 11, 890-897.

Johnson, K. L. and Tassinary, L. G. 2007. Compatibility of basic social perceptions determines perceived attractiveness. Proceedings of the National Academy of Sciences 104, 12, 5246-5251.

Kozlowski, L. And Cutting, J. 1977. Recognizing the sex of a walker from a dynamic point-light display. Perception and Psychophysics 21, 578-580.

Kozlowski, L. AND Cutting, J. 1978. Recognizing the gender of walkers from point-lights mounted on ankles: Some second thoughts. Perception and Psychophysics 23, 459.

Mather, G. and Murdoch, L. 1994. Gender discrimination in biological motion displays based on dynamic cues. Proceedings of the Royal Society of London, Series B 258, 273-279.

Troje, N. F. 2002. Decomposing biological motion: A framework for analysis and synthesis of human gait patterns. Journal of Vision 2, 5, 371-387.

Vinayagamoorthy, V., Gillies, M., Steed, A., Tanguy, E., Pan, X., Loscos, C., and Slater, M. $2006 . \quad$ Building expression into virtual characters. Eurographics State of the Art Reports.

Received December 2007; revised April 2008; accepted June 2008 